\title{
The Study of Depot Position Effect on Travel Distance in Order Picking Problem
}

\author{
Agung Chandra ${ }^{1}$ and Christine Natalia ${ }^{2}$ \\ 1 Department of Industrial Engineering, Universitas Mercu Buana, Indonesia \\ E-mail: 〈agung.chandra@mercubuana.ac.id〉 \\ ${ }^{2}$ Department of Industrial Engineering, Universitas Katolik Indonesia Atma Jaya, Indonesia \\ E-mail: 〈chrisnatalia@atmajaya.ac.id〉
}

\begin{abstract}
Research of travel distance on single - depot position in warehouse is tremendous. This study focuses more on the effect of two-depot position on travel distance in order picking problem (OPP) by using the concept of traveling salesman problem (TSP) and exact method - Branch and Bound (B\&B) algorithm. The total distance of one-depot position is shorter than two-depot position for single and double block warehouses and the difference is less than $5 \%$. The total distance is also compared with approximate methods - SA and TS which show that the differences are less than $5 \%$. The sequence of location visit for one depot and two depot is similar about two third from the total location visits. For order picking problem that has more than 25 location visits, one need to consider to apply approximate approach to get the solution faster even the difference will be higher from exact approach when the number of location visit or aisle increases.
\end{abstract}

Keywords: approximate approach, depot position, exact approach, order picking problem, travel distance

Received: September 23, 2021; accepted: December 6, 2021; available online: December 23, 2021

DOI: $10.17535 /$ crorr.2021.0016

\section{Introduction}

In the early year 2020, market competition becomes tougher on the global economy, affecting the financial and supply chain operations [1]. During this era, companies have been seeking the way to cope and deal with this condition, not only in finance but also operations. Efficiency and effectiveness are the keys to mitigate this competition. Cutting operations costs by downsizing labor, preventing unnecessary overtime, closing unprofitable business outlets, decreasing trucking rents, rescheduling supplier's payment, and debt restructuring is a common strategy for most companies to increase efficiency. This strategy must be optimized in order to achieve an ideal condition and harmonized working environment. One of the supply chain operations is warehousing activities. The main warehouse activities include receiving, transfer and put away, order picking / selection, accumulation / sortation, cross-docking and shipping. Order picking, which is the process of retrieving products from storage or buffer areas in response to a specific customer request, is the most labor intensive operation in warehouses with manual systems and a very capital intensive operation in warehouses with automated systems. The cost of order picking reaches $55 \%$ of the total operating expense in warehouse [2]. There are five main activities in order picking: to set up, pick, search, travel, and others such as obtaining the pick list and emptying the pick carrier. Traveling activity consumes about $50 \%$ of the total order-picking time $[3,4]$. Reducing order picking time means increasing efficiency. The efficiency of the order picking process depends on the size of warehouse, picking systems, 
storage strategies, layout strategies and routing strategies. The layout strategy covers place of the depot, number of blocks, size of the aisles, standard and non-standard layout configuration and rack combinations. The depot strategy analysis shows that the most effective strategy is picking in a middle depot and the combined route strategy is the best one in Very Narrow Aisle (VNA) $[5,6,7]$ concluded that the best depot location is in the middle of the front cross aisle and valid for several routing heuristics under random storage conditions. The chosen routing strategy has a direct impact on the length (distance) and travel time of the tour. Good routing strategies have successfully proved that it can decrease travel time around $30 \%$. Reduction of the travel (walking or driving) distance has a significant impact on the total order picking time [8]. Those studies mentioned above focus on a single depot in a warehouse. In this paper, the research objective is to study the effect of two depot positions on travel distance, then the results are compared to single depot position in order picking problem. One position occurs when receiving and shipping dock are at the same location (one depot) and the other position occurs when receiving and shipping docks are in the opposite locations (two depots). Depots are located at receiving and shipping areas because both the receiving and shipping areas of a warehouse are critical to its successful operation [9]. The TSP concept and optimization method - B\&B algorithm will be applied to this OPP study.

\section{Methodology and data}

\subsection{Description of Problem Instance}

The study was conducted on two different positions of depots in one block and two blocks warehouses in which the pick locations were randomly chosen. The warehouse was made of narrow vertical aisles and horizontal cross aisles. The depots were located at receiving and shipping areas, so that the following conditions occured. Receiving and shipping areas were located at the same dock, thus the depots were at the same dock too. The position of the depot was in the middle. In this case, the picker entered and exited through the same depot. See Figure 1. This is called U-flow configuration.

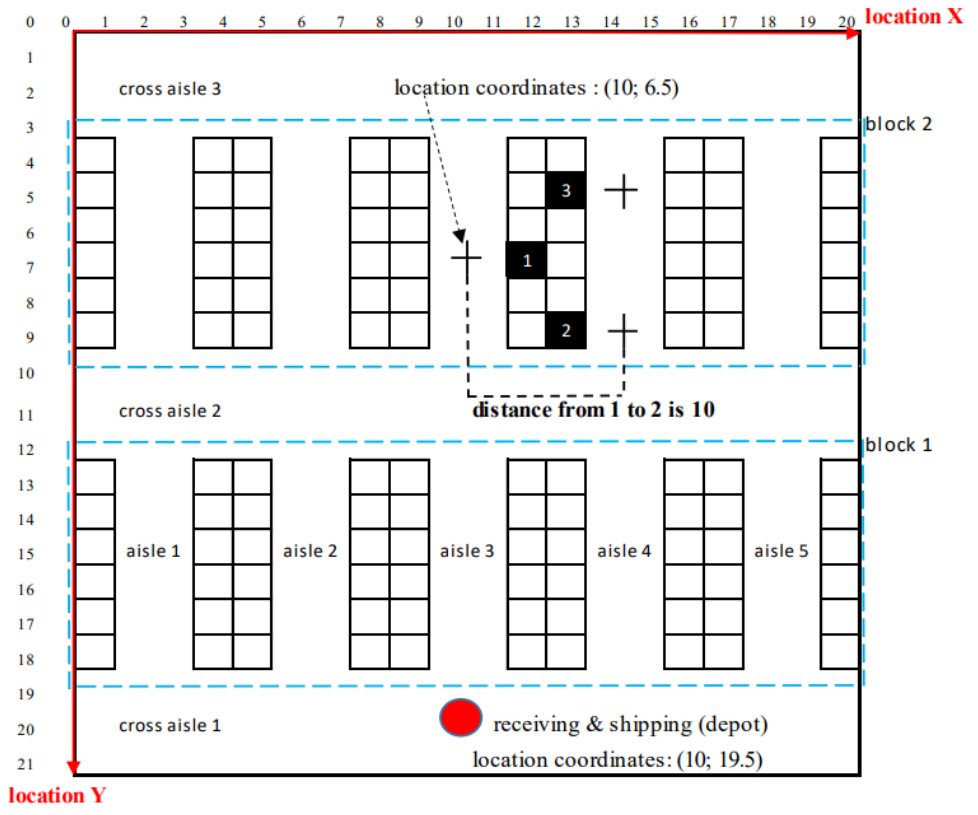

Figure 1: Receiving and Shipping docks are at the same dock (one depot) 
The receiving and shipping areas were located at the opposite docks, then one depot was at the receiving area and another one was at the shipping area. In this case, the picker started at the receiving depot, picked the items, then exited at the shipping depot. Both receiving and shipping positions were in the middle, see Figure 2. This is called flow-through configuration. The effects of these depot positions on travel distance were tested with the same pick list that had the same block, number of pick locations, and position. Hence, the position of the depot that had shorter distance would be found.The total picking location in this study was 100 .

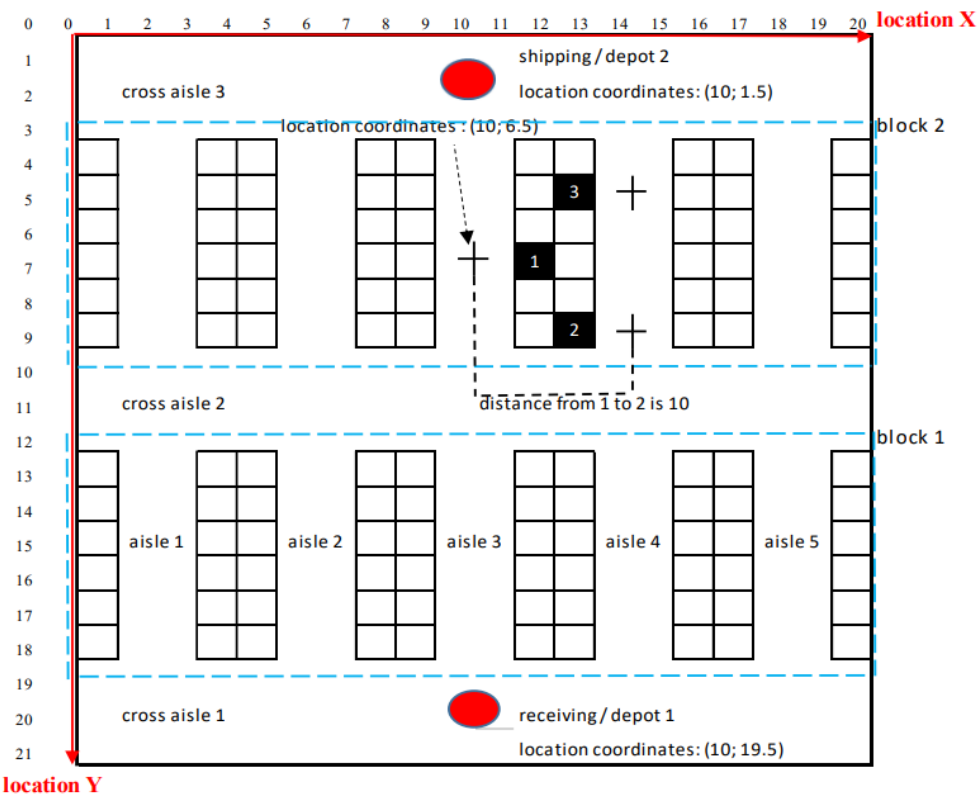

Figure 2: Receiving and Shipping docks are in opposite position (two depots)

The total distance was calculated by measuring distance between picking locations. TSP concept was applied [10] and optimization methods - exact approach was used to get the shortest distance. The TSP was OPP; the salesman was the order picker who was responsible to pick the products from their locations according to the picking list; and cities or towns were items or products to collect. Each square indicates picking location and the width was 1; aisle width was 2 ; cross aisle width was 3 . The length of each aisle was the same whilst the number of aisles were varied in this study.

\subsection{Application of TSP on OPP}

In the literature, TSP is classified as a NP-hard problem; likewise, TSP and Order Picking are closely related. A picking problem is defined by the warehouse - picking location and picking list. Picking list is a set of products described by their locations in the warehouse. Manual order picking has mainly focused on economic performance measures such as the shortest distance and time. Increasing economic performance can be done by robots, but not applicable in small to medium size companies. Then the purpose of routing picking is to get the shortest and most efficient picking $[11,12,13]$. 


$$
\begin{gathered}
\text { OF }=\text { Min } \sum_{i=1}^{n} \sum_{j=1}^{n} d_{i j} x_{i j} \\
\sum_{j=1}^{n} \quad x_{i j}=1 ; i=1, \cdots, n \\
\sum_{i=1}^{n} \quad x_{i j}=1 ; j=1, \cdots, n \\
x_{i j}=0 \text { or } 1
\end{gathered}
$$

Where $O F$ is Objective Function; $d_{i j}$ is distance from $i$ to $j$; in this study, $d$ is the distance from picking location $i$ to picking location $j ; n$ is the number of picking locations and $X_{i j}$ is binary variables. Since $X_{i j}$ can take only 0 or 1 , then this problem has a finite number of solution [14].

\subsection{Exact Approach - B\&B Algorithm}

The TSP : given a list of cities and distance matrix from each city to the other cities, then find the shortest distance that visits each city only once and returns to the starting city. The methods for solving the TSP usually can be divided into three basic components: a starting point, a solution generation scheme, and a termination rule. When the termination rule is such that the iteration stops if and only if a tour is optimal, then the method is exact. The branch and bound algorithm for the traveling salesman problem uses a branch and bound tree, like the branch and bound algorithms for the knapsack problem and for solving integer programs. $\mathrm{B} \& \mathrm{~B}$ algorithm is a fundamental and widely used methodology for producing exact or optimal solutions to NP-hard optimization problems. It creates and prunes the nodes, and this happens in a recursive way. The B\&B finds out all possible optimum solutions, and the minimum cost of the city is branched [15]. This algorithm has three main components: branching, bounding and selecting the next node. The pseudocode for the branch and bound algorithm is listed in Figure 3 below [16]:

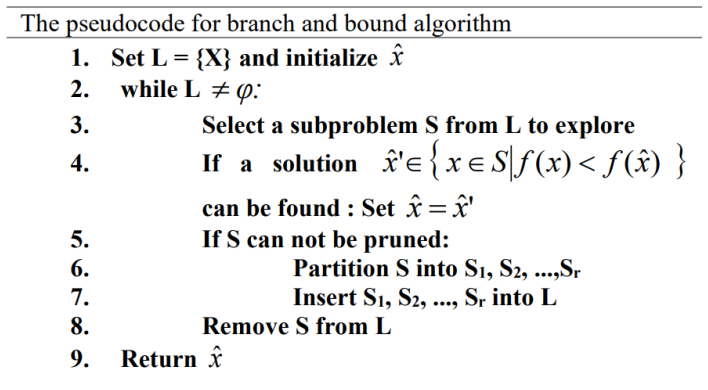

Figure 3: Pseudocode for BEBB algorithm.

To solve the optimization problem as $\mathrm{P}, \mathrm{B} \& \mathrm{~B}$ iteratively builds a search tree $\mathrm{T}$ of subproblems. Thus, the subsets of the search space set a list L of unexplored problems (line 1) and initialize the incumbent solution. Then, a subproblem S from L to explore is selected (line 3). At each iteration, the algorithm selects a new subproblem $S \subseteq X$ to explore from a list $\mathrm{L}$ of unexplored sub problems; if a solution (called a candidate incumbent) can be found with a better objective value than (i.e., the incumbent solution is updated. On the other hand, if it 
can be proven that no solution in $S$ has a better objective value than (i.e., $\forall x \in S, f(x) \geq f()$ ), the subproblem is pruned (or fathomed), and it is terminal (line 4 and 5). Branching strategy affects the number of children and partitions the way the subproblem do (line 6). Otherwise, child subproblems are generated by partitioning $S, S 1, S 2, \ldots, S r$, which are then inserted into T into an exhaustive (but not necessarily mutually exclusive) set of subproblems. Once no unexplored subproblem remains, the best incumbent solution is returned; since subproblems are only fathomed if they contain no solution better than, it must be the case that $\in \arg \min x \in X f(x)$.

\subsection{Framework of research}

The following Figure 4 is a framework to do the research which focuses on the comparison of total distance between warehouses with one depot and two depots by applying TSP on OPP. To calculate the total distance, one needs to create the symmetric distance matrix $n+1$ or $n+2$ containing minimal distance between two picking locations or between picking location and the depot. Matrix $n+1$ is for one depot and matrix $n+2$ is for two-depots warehouse where $\mathrm{n}$ is the number of picking locations in a pick list. The followings are some useful terminologies $[17,18]$ :

- A block contains all locations between two cross aisles.

- A sub aisle contains all items of one aisle in one block and the two intersections surrounding them.

- A depot is input / output point where in one depot condition, a picker starts and ends at the same depot; and in two-depot condition, a picker starts at one depot and ends at another depot.

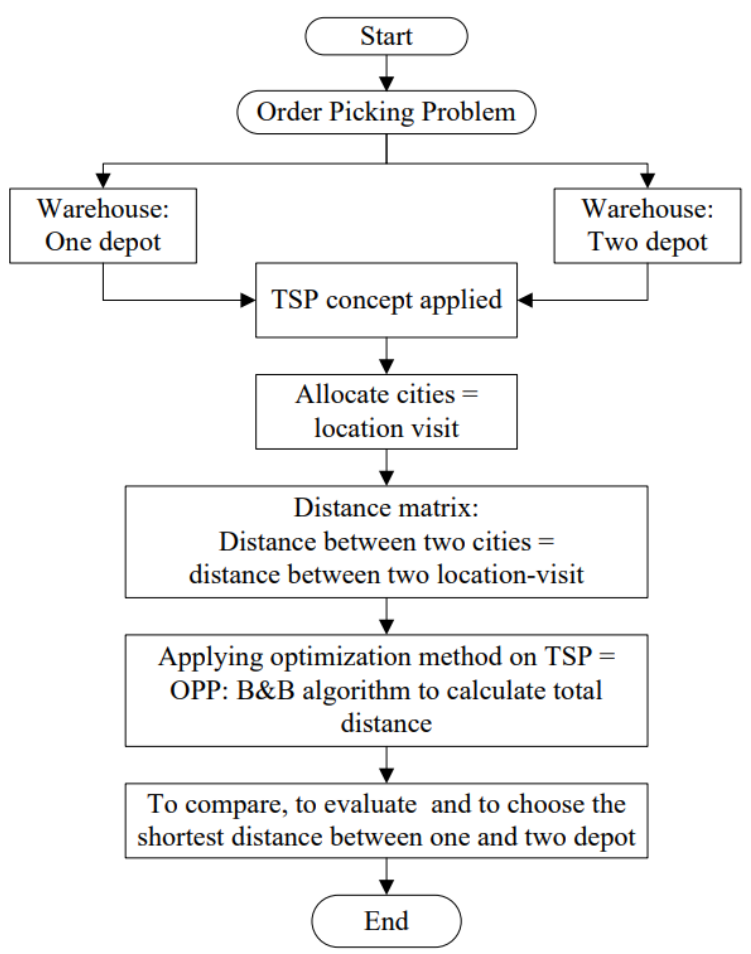

Figure 4: Framework of Research 
In this study, the researches assume that:

1. One pick list is done by one picker.

2. One stock keeping unit (SKU) is placed on one picking location.

3. Every pick list consist of SKU where location visit are randomly selected.

4. The position of depot is in the middle of side.

5. The picker walks in the middle of aisle and when the picker is picking, the position of the picker is in the centre of pick location.

6. The distance between two locations is the shortest distance even there are more than one alternatives

7. The distance from point $\mathrm{i}$ to point $\mathrm{j}$ is the same with the distance from point $\mathrm{j}$ to point $\mathrm{i}$, in other words the TSP is a symmetric TSP.

The effects of depot position were studied on one block and two block warehouses with various aisles and pick locations. For the pick locations, the picking time was limited to 30 because of time consideration [19].

\section{Results and Discussion}

The performance of the distance between one depot and two depots is processed by computer system i5, 8GB, x64 based processor and by using exact algorithm - B\&B algorithm in Lingo 18.0 program; then the results are compared as follows:

\begin{tabular}{|l|l|c|c|c|}
\hline Warehouse & Depot & Aisles & Location visit & Distance \\
\hline \multirow{5}{*}{ One Block } & & 5 & 15 & 91 \\
& \multirow{3}{*}{ One Depot } & 5 & 30 & 94 \\
& & 10 & 15 & 105 \\
& & 10 & 30 & 140 \\
\cline { 2 - 5 } & \multirow{3}{*}{ Two Depots } & 5 & 15 & 91 \\
& & 5 & 30 & 35 \\
& & 10 & 15 & 106 \\
& \multirow{3}{*}{ One Depot } & 5 & 30 & 144 \\
\cline { 2 - 5 } & \multirow{2}{*}{ Two Depots } & 5 & 25 & 160 \\
& & 10 & 25 & 170 \\
\hline \multirow{2}{*}{ Two Deks } & 5 & 25 & 162 \\
\hline
\end{tabular}

Table 1: Distance comparison between one depot and two depot OPP

From Table 1, it can be seen that when the number of aisles increases and the number of location visits are stable, the distance increases. Similarly, when the number of aisles is stable but the location visits increase, the distance increases as well. In other words, the distance increases when the number of aisles or the number of location visits increases. This happens for both one block and two block warehouses.

From Table 2 and Figure 5, the distance of one depot for order picking is compared to two depots. The difference between them is less than $5 \%$. The difference increases when the number of aisles or location visits increases. 
The Study of Depot Position Effect on Travel Distance in Order Picking Problem

\begin{tabular}{|l|c|c|c|c|c|}
\hline \multirow{2}{*}{ Warehouse } & \multirow{2}{*}{ Aisles } & \multirow{2}{*}{ Location visit } & \multicolumn{3}{|c|}{ Distance } \\
\cline { 4 - 6 } & & & One depot & Two depot & Difference(\%) \\
\hline \multirow{3}{*}{ One Block } & 5 & 15 & 91 & 91 & 0.00 \\
& 5 & 30 & 94 & 95 & 1.06 \\
& 10 & 15 & 105 & 106 & 0.95 \\
& 10 & 30 & 140 & 144 & 2.85 \\
\hline \multirow{2}{*}{ Two Blocks } & 5 & 25 & 160 & 162 & 3.33 \\
& 10 & 25 & 170 & 178 & 4.71 \\
\hline
\end{tabular}

Table 2: Distance gap between one depot and two depots

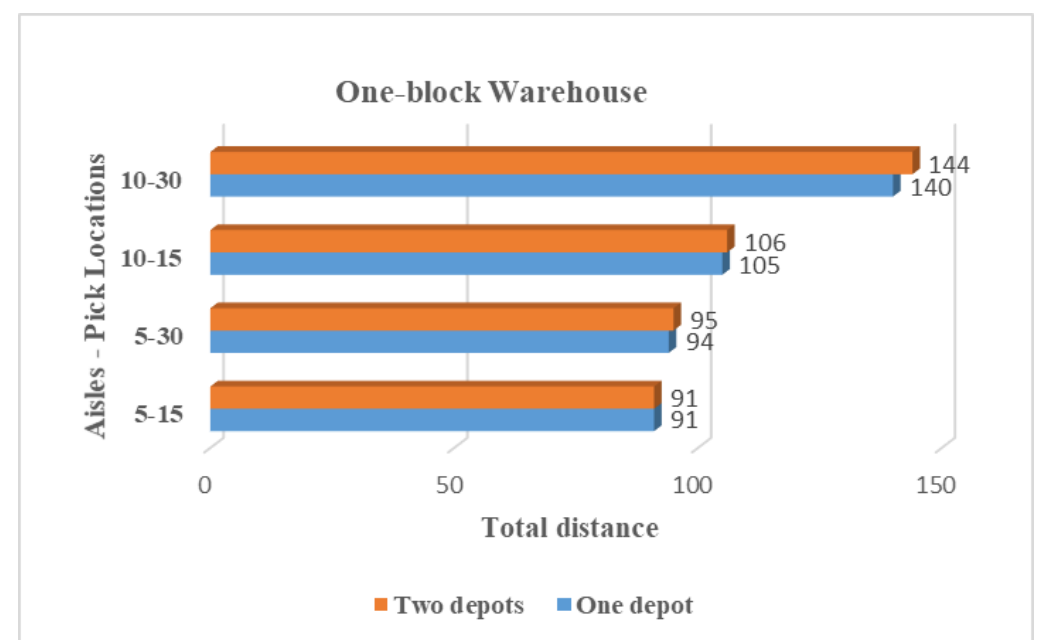

Figure 5: Aisles, Pick Locations, Total Distance in One Block Warehouse

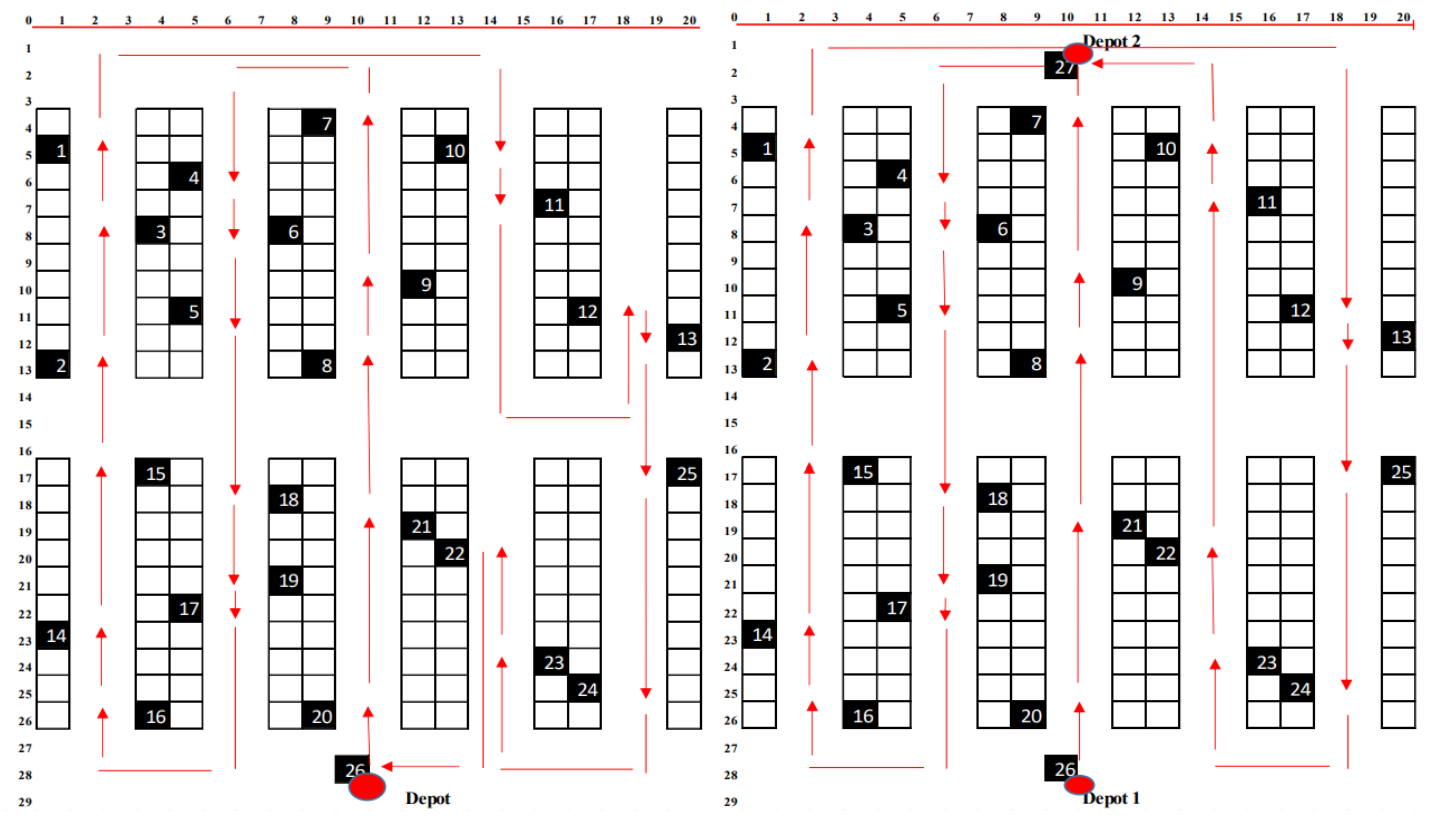

Figure 6: Flow of Two Block One Depot

Figure 7: Flow of Two Depots Warehouse 
As the distance between one depot and two depots are found and compared, the sequence between them were studied. The sequence and the total length are as follows:

- Two blocks, one depot warehouse (Figure 6): $26-20-21-8-9-7-4-6-5-18-$ $19-17-16-14-15-2-3-1-10-11-12-13-25-24-23-22-26=160$

- Two blocks, two depots warehouse (Figure 7): $26-20-21-8-9-7-4-6-5-18-$ $19-17-16-14-15-2-3-1-12-13-25-24-23-22-11-10-27=162$

The sequence for picking in a two-depots position is not much different than that in one-depot. In this case, only one-third of total location visits are different. In this study, one has to choose the shorter one, then one depot is better than two depots, even it does not exceed more than $5 \%$. Practically, most media and small enterprises implement the S-shape routing for picker and yield in distance 171 for one-depot two block and 162 for two-depot two block warehouses in distance. This is because S-shape is easy to execute. The routing itself tends to be a combined routing method. This route has a clear pattern which may reduce the time spent by order pickers on searching for locations and reduce the risk of pick errors as well.

In some cases, one pick list may have more than 30 location visits or whether 60 or 80 location visits, especially in the peak season then the time needed to solve the problems is not convenient anymore by the exact approach, because it needs hours per case. It will be more economical to use the approximate method - heuristics algorithm such as simulated annealing (SA) and tabu search (TS). Table 3 and 4 show us distance and time process comparison between $\mathrm{B} \& \mathrm{~B}, \mathrm{SA}$, and TS:

\begin{tabular}{|c|c|c|c|c|c|c|}
\hline \multirow{2}{*}{ Warehouse } & Aisles - Loc. to & B\&B & \multicolumn{2}{|c|}{ SA } & \multicolumn{2}{c|}{ TS } \\
\cline { 2 - 6 } & be picked & Distance & Distance & $\%$ Diff & Distance & $\%$ Diff \\
\hline \multirow{3}{*}{ One Block } & $5-15$ & 91 & 92 & $1.10 \%$ & 91 & $0.00 \%$ \\
\cline { 2 - 6 } & $5-30$ & 94 & 105 & $11.70 \%$ & 101 & $7.45 \%$ \\
\cline { 2 - 6 } & $10-15$ & 105 & 105 & $0.00 \%$ & 107 & $1.90 \%$ \\
\hline \multirow{2}{*}{ Two Blocks } & $10-30$ & 140 & 144 & $2.86 \%$ & 144 & $2.86 \%$ \\
\cline { 2 - 6 } & $10-25$ & 160 & 166 & $3.75 \%$ & 166 & $3.75 \%$ \\
\hline
\end{tabular}

Table 3: Distance comparison between Exact (B\&B) and Heuristics Approach (SA \& TS)

\begin{tabular}{|c|c|c|c|c|c|c|}
\hline \multirow{2}{*}{ Warehouse } & $\begin{array}{c}\text { Aisles - Loc. to } \\
\text { be picked }\end{array}$ & B\&B & \multicolumn{2}{|c|}{ SA } & \multicolumn{2}{c|}{ TS } \\
\cline { 2 - 6 } & Time $(\mathrm{s})$ & Time $(\mathrm{s})$ & $\%$ Diff & Time $(\mathrm{s})$ & $\%$ Diff \\
\hline \multirow{3}{*}{ One Block } & $5-15$ & 3.84 & 28.5 & $642.19 \%$ & 20.22 & $426.56 \%$ \\
\cline { 2 - 7 } & $5-30$ & 6562 & 30.72 & $-99.53 \%$ & 63.67 & $-99.03 \%$ \\
\cline { 2 - 7 } & $10-15$ & 0.33 & 23.22 & $6936.36 \%$ & 17.27 & $5133.33 \%$ \\
\hline \multirow{2}{*}{ Two Blocks } & $10-30$ & $>86,400$ & 30.13 & - & 62.53 & - \\
\cline { 2 - 7 } & $10-25$ & 1.98 & 26.77 & $1252.02 \%$ & 44.73 & $2159.09 \%$ \\
\hline
\end{tabular}

Table 4: Time process comparison between Exact (B\&B) and Heuristics Approach (SA \& TS)

For the pick list which has more than 25 location visits, it is more beneficial in time to use approximate method than the exact method. Meanwhile, for the distance, the results of SA are different from exact method around $4.4 \%$ on average and the TS around $2.7 \%$. In this case, TS algorithm is better than SA. The rank for each method in both distance and time is: 
- Distance from the shortest to the longest: B\&B - TS - SA

- Time from the fastest to the slowest: $\mathrm{SA}-\mathrm{TS}-\mathrm{BB}$

To minimize the distance and time spent, one can also place SKU that has a lot of movement of frequently visits at the best locations according to the depot position one has, or in other words, the greatest convenient locations, as seen in the following figure which are remarked with darker colour:
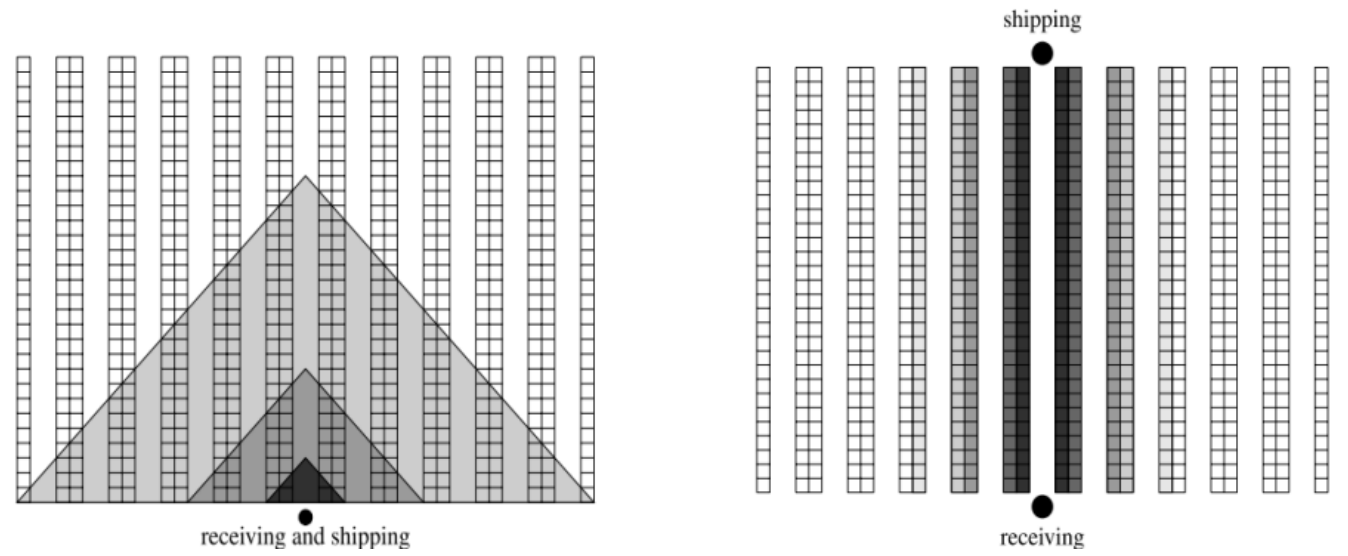

Figure 8: More convenient locations are in one depot and two depots remarked by darker colour [20]

\section{Conclusion}

Many researchers have been focusing on the travel distance of single depot warehouses, but some enterprises have two-depot warehouses. In this paper, the effects of two-depot position on travel distance have been studied. By using TSP concept and exact method - B\&B algorithm, the findings indicate that total distance for order picking problem in one depot is shorter than two depots both for one block and two blocks warehouses, and the difference is less than $5 \%$. The sequences of location visits for one depot and two depots are similar, which are about two thirds from the total location visits. The total distance is also compared using approximate methods - SA and TS which show that the differences are less than 5\%. For the order picking problem that has more than 25 location visits, one needs to consider applying an approximate method to get the solution faster as the difference becomes higher when the number of location visits or aisles increases. For further research, the study can be extended to multiple pickers and three-block warehouses to validate these findings.

\section{Acknowledgements}

The authors would like to acknowledge support of materials and guidances from Prof. Dr. Aurelija Burinskiene from Vilnius Gediminas Technical University, Lithuania.

\section{References}

[1] Wu, D.D. and Olson, D.L. (2020). Pandemic Risk Management in Operations and Finance: Modeling the Impact of Covid19. Springer.

[2] De Koster, R., Le-Duc, T., and Roodbergen, K.J. (2007). Design and Control of Warehouse Order Picking: A Literature Review. European Journal of Operational Research 182(2), 481-501. 
[3] Dukic, G. and Oluic, C. (2007). Order-Picking Methods: Improving Order-Picking Efficiency. International Journal of Logistics System and Management 3(4), 451- 460.

[4] Roodbergen, K.J. and De Koster, R. (2001). Routing Methods for Warehouse with Multiple CrossAisles. International Journal of Production Research 39 (9), 1865-1883.

[5] Burinskiene, A. (2010). Order Picking Process at Warehouse. International Journal of Logistics Systems and Management 6 (2), 162 - 178. doi: 10.1504/IJLSM.2010.030958

[6] Bassan, Y., Roll, Y., and Rosenblatt, M.J. (1980). Internal Layout Design of a Warehouse. AIIE Transaction 12(4), $317-322$.

[7] Roodbergen, K.J. and Vis, I.F.A. (2006). A Model for Warehouse Layout. IIE Transactions 38(10), $799-811$.

[8] De Koster, R., Roodbergen, K.J., and van Voorden, R. (1999). Reduction of Walking Time in the Distribution Center of De Bijenkorf. In Speranza, M.G. and Stahly, P. (eds.) New Trends in Distribution Logistics (pp.215-2134). Springer, Berlin.

[9] Rushton, A., Croucher, P., and Baker, P. (2014). The Handbook of Logistics and Distribution Management. Kogan Page.

[10] Pansart, L. (2016). Research Project: Exact Algorithm for Picking Problem. Universite Grenoble Alpes.

[11] Calzavara, M., Glock, C., Grosse, E., Persona, A., \& Sgarbossa, F. (2017). Analysis of economic and ergonomic performance measures of different rack layouts in an order picking warehouse. Computers \& Industrial Engineering, 111, 527-536. doi: 10.1016/j.cie.2016.07.001

[12] Bottani, E., Casella, G., Montanari, R. (2018). Distance Estimate for Order Picking Systems in Manual Warehouse. In Proceedings of the International Conference on Harbor Maritime and Multimodal Logistics M\&S, Bottani, Bruzzone, Longo, Merkuryev, and Piera eds.

[13] Madani, A., Batta, R., and Karwan, M. (2021). The Balancing Traveling Salesman Problem: Application to Warehouse Order Picking. TOP29, p.442-469. doi: 10.1007/s11750-020-00557-y

[14] Mataija, M., Rakamarić Šegić, M., Jozić, F. (2016). Solving the travelling salesman problem using the Branch and Bound Method. Zbornik Veleučilišta u Rijeci, Vol. 4 No. 1, pp. 259-270

[15] Karthy, T. and Priyanka, S. (2019). Comparative Analysis of the Optimal Solutions of Travelling Salesman Problem, AIP Conference Proceeding 2112, 020030. doi: 10.1063/1.5112215

[16] Morrison, D., Jacobson, S.H., Sauppe, J.J., Sewell, E.C. (2016), Branch and Bound algorithms: A Survey of Recent Advances in Searching, Branching, and Pruning, Discrete Optimization (19), p.79-102. DOI: doi: 10.1016/j.disopt.2016.01.005

[17] Masae, M. (2019). Developing Efficient Order Picker Routing Policies in Manual Picker-to-Parts Order Picking Systems. Dissertation at Technische Universitat Darmstadt.

[18] Pansart, L., Catusse, N., Cambazard, H. (2018). Exact Algorithms for the Order Picking Problems. Computers and Operations Research. Elsevier. doi: 10.1016/j.cor.2018.07.002. Hal-01481915v3

[19] Chandra, A. and Natalia, C. (2021). Performance Analysis of Optimization Methods for Solving Traveling Salesman Problem. Innovative Technologies and Scientific Solutions for Industries no.1(15), p.69 -75. doi: 10.30837/ITSSI.2021.15.069

[20] Bartholdi, III, J.J and Hackman, S.T. (2014). Warehouse and Distribution Science R.096. The Supply Chain and Logistics Institute. School of Industrial and System Engineering, Georgia Institute of Technology, Atlanta, U.S.A. Available at www.warehouse-science.com. 